\title{
Data Assimilation for Navier-Stokes using the Least-Squares Finite-Element Method
}

\author{
Alexander Schwarz \\ Institut für Mechanik, University of Duisburg-Essen, Universitätsstraße 15, 45117 Essen, Germany.
}

Richard P. Dwight

Aerodynamics Group, Faculty of Aerospace, TU Delft, P.O. Box 5058, 2600GB Delft, The Netherlands.

\begin{abstract}
We investigate theoretically and numerically the use of the Least-Squares Finite-element method (LSFEM) to approach data-assimilation problems for the steady-state, incompressible Navier-Stokes equations. Our LSFEM discretization is based on a stressvelocity-pressure (S-V-P) first-order formulation, using discrete counterparts of the Sobolev spaces $H(\mathrm{div}) \times H^{1} \times L^{2}$ for the variables respectively. In general S-V-P formulations are promising when the stresses are of special interest, e.g. for non-Newtonian, multiphase or turbulent flows. Resolution of the system is via minimization of a leastsquares functional representing the magnitude of the residual of the equations. A simple and immediate approach to extend this solver to data-assimilation is to add a datadiscrepancy term to the functional. Whereas most data-assimilation techniques require a large number of evaluations of the forward-simulation and are therefore very expensive, the approach proposed in this work uniquely has the same cost as a single forward run. However, the question arises: what is the statistical model implied by this choice? We answer this within the Bayesian framework, establishing the latent background covariance model and the likelihood. Further we demonstrate that - in the linear case - the method is equivalent to application of the Kalman filter, and derive the posterior covariance. We practically demonstrate the capabilities of our method on a backward-facing step case. Our LSFEM formulation (without data) is shown to have good approximation quality, even on relatively coarse meshes - in particular with respect to mass-conservation and reattachment location. Adding limited velocity measurements from experiment, we show that the method is able to correct for discretization error on very coarse meshes, as well as correct for the influence of unknown and uncertain boundary-conditions.
\end{abstract}

Keywords: Least-squares finite element method, data assimilation, inverse problems, fluid dynamics, Navier-Stokes, Bayesian inference, uncertainty quantification 


\section{Introduction}

Increasingly there is a demand for numerical methods for fluids capable of assimilating experimental data from wind-tunnel measurements into CFD simulation. This can be done with several goals: identifying unmeasured variables such as pressure from particleimage velocimetry (PIV) velocity field measurements [1]; increasing the time-resolution of measurement data 22; filling spatial gaps in PIV data 3]; or filtering noisy data 4 . The methods used may be seen as physics-based filtering/regression techniques. In each case the ability of a simulation to smoothly approximate the flow-field in the full domain in all variables, or identify an unmeasured quantity of interest, increases the value of the measurement. An increase in the amount of data provided by modern experimental techniques drives a trend toward increasing demand for effective post-processing tools, with an increasing amount of physical fidelity [5, 2, 3,

In this paper we investigate an approach to this problem for assimilating experimental velocity measurements into Navier-Stokes flow based on a Least-Squares Finite-Element Method (LSFEM) [6, 7]. Briefly: given a system of PDEs with boundary conditions, LSFEM methods define a scalar functional whose minimum is the solution of the system. The functional is defined as a sum of e.g. $L^{2}$-norms of the residuals of the original PDE and optionally BCs. These terms are then discretized with finite elements spaces. By construction at least one minimum of the functional exists, even when the original PDE plus BCs is ill-posed in the sense of Hadamard. Since the discretization is already constructed in a least-squares sense, to add information from experimental data, we only have to add a term quadratically penalizing the difference between predictions and observations. The weighting of this term determines the extent to which the solution will match the observations and is chosen in inverse proportion to the measurement accuracy.

The method (which we refer to as LSFEM+) was first proposed for Navier-Stokes by Heyes et al. [8] and later investigated by the present authors 9. It has desirable theoretical behaviour in the limits: in the absence of data we retrieve the standard LSFEM flow solution, and for plentiful, perfectly accurate data we interpolate the data exactly. Furthermore LSFEM+ is unusual in being capable of data assimilation at a computational cost equivalent to a single flow simulation, see Section 1.1 .

The accuracy of the method will depend on the quality of the underlying LSFEM discretization in regions in which no data is available. Our aim in this paper is to provide a mixed LSFEM with improved accuracy (also with respect to mass conservation), but without additional variables or degrees of freedom. Starting from the incompressible Navier-Stokes equations we derive a div-grad first-order system resulting in a three-field approach with stresses, velocities, and pressure as unknowns. In our formulation the stresses are approximated by using shape functions related to the edges. These quadratic vector-valued functions are used for the interpolation of the rows of the stress tensor and belong to a Raviart-Thomas space, which guarantees a conforming discretization of the Sobolev space $H(d i v)$. Furthermore, standard cubic and linear polynomials associated to the vertices of the triangle are used for the continuous approximation of the velocities and the pressure respectively. This approach is similar that of Cai et al. [10] who developed theoretical properties of this discretization for Stokes flow.

In summary, the contributions of this paper are: firstly to extend the stress-velocitypressure formulation to Navier-Stokes, and perform a numerical investigation of a real experiment. Secondly to show the effectiveness and efficiency of data assimilation with 
this LSFEM approach, including solving inverse problems with uncertain and unknown boundary conditions, at a computational cost equivalent to a single solve of the forward problem. Thirdly to motivative the choice of data weighting based on measurement error in a Bayesian statistical framework.

The paper is outlined as follows: in Section 1.1 we give a short overview of alternative data assimilation approaches, and in Section 1.2 a brief overview of LSFEM methods for fluids. The stress-velocity-pressure formulation is derived in Section 2. The Bayesian derivation and analysis of the added-data method is given in Section 3 . The method is numerically evaluated for a laminar backward-facing step in Section 4 , and conclusions are given in Section 5 .

\subsection{Data Assimilation Context}

As mentioned LSFEM+ has a cost of a single forward flow simulation. In contrast the computational cost of some standard stochastic data assimilation methods is much higher: Ensemble Kalman filtering costs typically 100 flow solves [11, 12]; 4D-Var requires about 50-100 flow and adjoint solves for nonlinear problems; physics based Reduced Order Model fitting (ROMs) requires training the ROM on the parameter space of interest, for rarely less than 100 solves 13,14 .

There are two other notable approaches with a comparable computational cost to our proposed method. The first is Optimal Interpolation, see e.g. [11, a minimum variance estimator. It is similar in form to the Kalman filter, in that it consists of a forecast step followed by an analysis step, except that the forecast step does not propagate the uncertainty through the model as in the Kalman filter, instead only the mean is propagated and fresh randomness is generated on the result according to a user-specified covariance structure. Thus the magnitude and covariance structure of the uncertainty due to the model is not preserved, and thus the method relies on a good guess of these. The second "cheap" approach is Measurement-Integrated Simulation [15, 16, in which a nonphysical forcing term is added to the Navier-Stokes equations. The forcing is designed to push the solution in the direction of experimental observations, and disappears in the case of exact experiment-simulation agreement. The strength of the forcing is an influential free parameter, having a similar effect to the Kalman gain in the Kalman filter, but currently the statistical interpretation of this approach is not clear.

\subsection{Least-Squares Finite-Elements for Incompressible Navier-Stokes}

In recent years the least-squares method was successfully applied to many problems in fluid dynamics and solid mechanics. The development and improvement of mixed leastsquares finite element formulations have been the objective of many studies. Exhaustive overviews with respect to theoretical foundations and application-oriented issues can be found for example in the monographs [17] and [18] and in the review [19].

Least-squares variational principles have increasingly gained attention, which is due to some theoretical and computational advantages compared to the Galerkin method. But nevertheless, the Galerkin method is today the most employed variational approach for all kind of boundary value problems in computational mechanics. The least-squares method in contrast only play a minor role. From the point of view of an engineer the reasons are evident: since the 1970s attention has focussed on low-order elements which 
are easy to implement, robust, and lead to sparse, well-conditioned stiffness matrices. Unfortunately, the approximation quality of low-order least-squares finite elements is moderate and consequently only little attention was paid to the method. An illustrative example for the poor solution of lower-order interpolation was given in 20 for the driven cavity problem. While it is possible to obtain qualitatively correct results, the method consistently needs more degrees of freedom compared to alternative methods. Despite this drawback, the least-squares method has also some important advantages [17, 18]

- The method provides an a posteriori error estimate without additional costs, which can be used for adaptive mesh refinement.

- The flexibility to design functionals directly approximating field variables of interest, e.g. stresses, vorticity, velocities.

- The method is not restricted by the LBB-condition regarding the choice of the polynomial degree of the finite element spaces.

- The resulting positive definite system matrices can be solved by using robust and fast iterative methods even for problems with non-self-adjoint operators.

These features yield the basis for theoretical analysis and numerical studies on e.g. ellipticity, error estimation, residual weighting and convergence and are investigated in the field of fluid dynamics by many authors, see e.g. 18 and the references therein. The utilized first-order systems in general consist of a composition of different quantities and could be divided into three approaches:

- The vorticity, velocity, pressure (VVP) formulation, see e.g. [17, 21] and 22].

- The stresses, velocity, pressure (SVP) formulation, see e.g. 23], 24] and 25].

- The velocity gradient, velocity, pressure (VGVP) formulation, see e.g. [26], 27] and [28.

The utilized first-order systems in general consist of a composition of the flow velocity, the gradient of the flow velocity, the pressure, the stresses, and the vorticity as dependent variables.

The main point of criticism of LSFEM in fluid-dynamics is the lack of numerical conservation, in particular mass conservation. Various strategies, e.g. mesh refinement, residual weighting, use of Lagrange multipliers, and improved velocity-pressure coupling have been used to mitigate this disadvantage somewhat [28, 29, 30, 31, 32. In recent works, spectral finite elements with higher-order polynomial finite element spaces $(p \geq 4)$ have demonstrated a satisfying approximation of the governing fields, with improved mass conservation 33, 34, 35]. Extensive work was done by the group from the University of Boulder [36, 37, 38, on the choice of first-order system, aiming to achieve stronger coupling between velocity and pressure, realised by introducing new variables depending on both fields, or by special treatment of the boundary conditions. The benefits achieved by all these techniques in combination allows acceptable performance of LSFEM for incompressible fluids. 


\section{LSFEM Discretization of Navier-Stokes}

In the following we describe the least-squares mixed finite element method for first-order linear and nonlinear systems, see e.g. [6, 7. A stress-velocity-pressure formulation for the incompressible Navier-Stokes equations is constructed, and finally we introduce a data term into the LSFEM functional. We work on the open bounded domain $\Omega \subset \mathbb{R}^{d}$ with piecewise-smooth boundary $\Gamma$.

\subsection{Linear PDEs}

Consider a (first-order) system of linear partial differential equations

$$
L w=f \text { on } \Omega, \quad B w=g \text { on } \Gamma,
$$

where $f \in L^{2}(\Omega), g \in L^{2}(\Gamma)$ and $L$ and $B$ denote linear differential operators on $\Omega$ and $\Gamma$ respectively. Let $(\cdot, \cdot)_{\Omega}$ and $(\cdot, \cdot)_{\Gamma}$ represent the $L^{2}(\Omega)$ and $L^{2}(\Gamma)$ scalar products. Also let $V$ be a suitable Sobolev space, then (1) can be reformulated as 6 .

$$
\min _{w \in V}[\underbrace{\left[\|L w-f\|_{\Omega}^{2}+\|B w-g\|_{\Gamma}^{2}\right]}_{J(w ; f, g)},
$$

where $\|\cdot\|_{\Omega}^{2}$ and $\|\cdot\|_{\Gamma}^{2}$ are the norms induced by the above scalar products, and $J$ is the least-squares functional.

The least-squares variational formulation is obtained by requiring that the first variation of $J$ vanishes at $w$ for all admissible perturbuations $\delta w \in V$ :

$$
\lim _{\epsilon \rightarrow 0} \frac{\mathrm{d}}{\mathrm{d} \epsilon} J(w+\epsilon \delta w)=0
$$

from which follows: Find $w \in V$ such that

$$
\underbrace{(L w, L \delta w)_{\Omega}+(B w, B \delta w)_{\Gamma}}_{Q(w, \delta w)}=\underbrace{(f, L \delta w)_{\Omega}+(g, B \delta w)_{\Gamma}}_{l(\delta w)}, \quad \forall \delta w \in V,
$$

where $Q(\cdot, \cdot)$ is a symmetric bilinear form and $l(\cdot)$ a linear form. This equation can be discretized by replacing $V$ with a discrete function space $V_{h} \subset V$. The resulting discretization can be shown to have a unique stable solution provided $L$ is bounded below [7] - in particular no equivalent of the LBB condition from mixed Galerkin formulations is required.

\subsection{Nonlinear PDEs}

Consider the nonlinear system of PDEs

$$
N w=0 \quad \text { on } \Omega, \quad B w=0 \quad \text { on } \Gamma,
$$

where $N$ is a nonlinear differential operator on $\Omega$, and $B$ is a nonlinear boundary operator. Assume we can find a suitable function space $V$ for solutions of this system. There are two options for formulating nonlinear LSFEM, we can either (a) linearize $N$, and build 
the LSFEM functional on the linearized governing equation, which then acts as a Newton iteration for the nonlinear system, or (b) build the functional directly on the nonlinear operator, and use Newton's method to find a root of the 1st-variation [39]. The latter results in an extra term in the 2nd-variation; and this is the approach we follow here.

The LSFEM formualtion is then

$$
\min _{w \in W}\left[\|N w\|_{\Omega}^{2}+\|B w\|_{\Gamma}^{2}\right]=\min _{w \in W} \underbrace{\left[(N w, N w)_{\Omega}+(B w, B w)_{\Gamma}\right]}_{\mathcal{J}(w)} .
$$

Taking 1st-variations gives

$$
\delta \mathcal{J}(w ; \delta w)=\left(N w, N^{\prime} \delta w\right)_{\Omega}+\left(B w, B^{\prime} \delta w\right)_{\Gamma},
$$

assuming that the Fréchet derivatives $N^{\prime}[w], B^{\prime}[w]$ of $N$ and $B$ about $w$ exist. Secondvariations results in

$$
\begin{aligned}
\Delta \delta \mathcal{J}(w ; \delta w, \Delta w) & =\left(N^{\prime} \Delta w, N^{\prime} \delta w\right)_{\Omega}+\left(N w, N^{\prime \prime} \Delta \delta w\right)_{\Omega} \\
& +\left(B^{\prime} \Delta w, B^{\prime} \delta w\right)_{\Gamma}+\left(B w, B^{\prime \prime} \Delta \delta w\right)_{\Gamma},
\end{aligned}
$$

where the final term is zero in the common case of linear $B$. Note that the term $\left(N w, N^{\prime \prime} \Delta \delta w\right)_{\Omega}$ is not present in the nonlinear formulation (a) mentioned above. Given these variations the Newton iteration for the update $\Delta w$ is:

$$
\Delta \delta \mathcal{J}(w ; \delta w, \Delta w)+\delta \mathcal{J}(w ; \delta w)=0 .
$$

\subsection{Navier-Stokes in $\sigma-v-p$ form}

In order to describe a boundary-value problem for Newtonian fluid flow, we consider the steady incompressible Navier-Stokes equations consisting of balances of momentum and mass

$$
\begin{aligned}
-\rho \nabla \boldsymbol{v} \boldsymbol{v}+2 \rho \nu \operatorname{div} \nabla^{s} \boldsymbol{v}-\nabla p & =\mathbf{0} \\
\operatorname{div} \boldsymbol{v} & =0 .
\end{aligned}
$$

Here, $\boldsymbol{v}$ denotes the velocities, $p$ the pressure, $\rho$ the density and $\nu$ the kinematic viscosity of a medium flowing through the domain. Furthermore,

$$
\nabla^{s} \boldsymbol{v}=\frac{1}{2}\left(\nabla \boldsymbol{v}+\nabla \boldsymbol{v}^{T}\right)
$$

denotes the symmetric velocity gradient. System (4) is of second order and can be transformed into a first-order div-grad system by means of an additional variable, namely the Cauchy stresses

$$
\boldsymbol{\sigma}=2 \rho \nu \nabla^{s} \boldsymbol{v}-p \mathbf{1} .
$$

We achieve

$$
\begin{aligned}
\operatorname{div} \boldsymbol{\sigma}-\rho \nabla \boldsymbol{v} \boldsymbol{v} & =\mathbf{0} \\
\boldsymbol{\sigma}-2 \rho \nu \nabla^{s} \boldsymbol{v}+p \mathbf{1} & =\mathbf{0} \\
\operatorname{div} \boldsymbol{v} & =0
\end{aligned}
$$

which is the basis for the nonlinear differential operator $N$, see e.g. [10. In order to complete the boundary value problem we split the boundary of the domain $\Gamma$ into two subsets, namely the Dirichlet boundary $\Gamma_{D}$ and Neumann boundary $\Gamma_{N}$ with the definitions

$$
\Gamma_{D} \cup \Gamma_{N}=\Gamma \quad \text { and } \quad \Gamma_{D} \cap \Gamma_{N}=\emptyset,
$$


on which the boundary conditions are

$$
\boldsymbol{v}=\boldsymbol{g} \quad \text { on } \Gamma_{D}, \quad p=q \quad \text { and } \quad \boldsymbol{\sigma} \boldsymbol{n}=\boldsymbol{h} \quad \text { on } \Gamma_{N} .
$$

The resulting quadratic least-squares functional for the set of equations (7) is given in terms of the $L_{2}$-norm as

$$
\mathcal{J}(\boldsymbol{\sigma}, \boldsymbol{v}, p)=\frac{1}{2}\left(\left\|\frac{1}{\sqrt{\rho}}(\operatorname{div} \boldsymbol{\sigma}-\rho \nabla \boldsymbol{v} \boldsymbol{v})\right\|^{2}+\left\|\frac{1}{\sqrt{\rho \nu}}\left(\boldsymbol{\sigma}-2 \rho \nu \nabla^{s} \boldsymbol{v}+p \mathbf{1}\right)\right\|^{2}+\|\operatorname{div} \boldsymbol{v}\|^{2}\right),
$$

where we have used some additional physically motivated weights on the 1st and 2nd terms, see e.g. [40].

The approximation spaces for $\mathcal{J}(\boldsymbol{\sigma}, \boldsymbol{v}, p): \boldsymbol{X} \times \boldsymbol{V} \times \boldsymbol{Q} \rightarrow \mathbb{R}$ are given by

$$
\boldsymbol{X}=\left\{\boldsymbol{\sigma} \in H(\operatorname{div}, \Omega)^{2}\right\}, \quad \boldsymbol{V}=\left\{\boldsymbol{v} \in H^{1}(\Omega)^{2}\right\} \quad \text { and } \quad \boldsymbol{Q}=\left\{p \in L_{2}(\Omega)^{2}\right\}
$$

The minimization of $\mathcal{J}$ presupposes the first variation is zero

$$
\delta_{\sigma, v, p} \mathcal{J}(\boldsymbol{\sigma}, \boldsymbol{v}, p, \delta \boldsymbol{\sigma}, \delta \boldsymbol{v}, \delta p)=0
$$

or explicitly:

$$
\begin{aligned}
\delta_{v} \mathcal{J}= & \int_{\Omega} \operatorname{div} \delta \boldsymbol{v} \cdot \operatorname{div} \boldsymbol{v} d V-\frac{1}{\rho \nu} \int_{\Omega} 2 \rho \nu \nabla^{s} \delta \boldsymbol{v} \cdot\left(\boldsymbol{\sigma}-2 \rho \nu \nabla^{s} \boldsymbol{v}+p \mathbf{1}\right) d V \\
& -\frac{1}{\rho} \int_{\Omega}(\rho \nabla \delta \boldsymbol{v} \boldsymbol{v}+\rho \nabla \boldsymbol{v} \delta \boldsymbol{v}) \cdot(\operatorname{div} \boldsymbol{\sigma}-\rho \nabla \boldsymbol{v} \boldsymbol{v}) d V=0 \\
\delta_{\sigma} \mathcal{J}= & \frac{1}{\rho} \int_{\Omega} \operatorname{div} \delta \boldsymbol{\sigma} \cdot(\operatorname{div} \boldsymbol{\sigma}-\rho \nabla \boldsymbol{v} \boldsymbol{v}) d V+\frac{1}{\rho \nu} \int_{\Omega} \delta \boldsymbol{\sigma} \cdot\left(\boldsymbol{\sigma}-2 \rho \nu \nabla^{s} \boldsymbol{v}+p \mathbf{1}\right) d V=0 \\
\delta_{p} \mathcal{J}= & \frac{1}{\rho \nu} \int_{\Omega} \delta p \mathbf{1} \cdot\left(\boldsymbol{\sigma}-2 \rho \nu \nabla^{s} \boldsymbol{v}+p \mathbf{1}\right) d V=0 .
\end{aligned}
$$

In order to seek the minimizer with Newton's method, we linearize $\delta \mathcal{J}$ with respect to stresses, velocities and pressure. Consequently, the second variation of $\mathcal{J}$ denoted as 
$\Delta \delta \mathcal{J}$, which is the basis for the Newton tangent, is explicitly

$$
\begin{aligned}
\Delta \delta_{v v} \mathcal{J}= & \int_{\Omega} \operatorname{div} \delta \boldsymbol{v} \cdot \operatorname{div} \Delta \boldsymbol{v} d V+\frac{1}{\rho \nu} \int_{\Omega} 2 \rho \nu \nabla^{s} \delta \boldsymbol{v} \cdot 2 \rho \nu \nabla^{s} \delta \boldsymbol{v} d V \\
& +\frac{1}{\rho} \int_{\Omega}(\rho \nabla \delta \boldsymbol{v} \boldsymbol{v}+\rho \nabla \boldsymbol{v} \delta \boldsymbol{v}) \cdot(\rho \nabla \Delta \boldsymbol{v} \boldsymbol{v}+\rho \nabla \boldsymbol{v} \Delta \boldsymbol{v}) d V \\
& -\frac{1}{\rho} \int_{\Omega}(\rho \nabla \delta \boldsymbol{v} \Delta \boldsymbol{v}+\rho \nabla \Delta \boldsymbol{v} \delta \boldsymbol{v}) \cdot(\operatorname{div} \boldsymbol{\sigma}-\rho \nabla \boldsymbol{v} \boldsymbol{v}) d V \\
\Delta \delta_{\sigma \sigma} \mathcal{J}= & \frac{1}{\rho} \int_{\Omega} \operatorname{div} \delta \boldsymbol{\sigma} \cdot \Delta \operatorname{div} \boldsymbol{\sigma} d V+\frac{1}{\rho \nu} \int_{\Omega} \delta \boldsymbol{\sigma} \cdot \Delta \boldsymbol{\sigma} d V \\
\Delta \delta_{p p} \mathcal{J}= & \frac{1}{\rho \nu} \int_{\Omega} \delta p \mathbf{1} \cdot \Delta p \mathbf{1} d V \\
\Delta \delta_{v \sigma} \mathcal{J}= & -\frac{1}{\rho \nu} \int_{\Omega} 2 \rho \nu \nabla^{s} \delta \boldsymbol{v} \cdot \Delta \boldsymbol{\sigma}-\frac{1}{\rho} \int_{\Omega}(\rho \nabla \delta \boldsymbol{v} \boldsymbol{v}+\rho \nabla \boldsymbol{v} \delta \boldsymbol{v}) \cdot \operatorname{div} \Delta \boldsymbol{\sigma} d V \\
\Delta \delta_{v p} \mathcal{J}= & -\frac{1}{\rho \nu} \int_{\Omega} 2 \rho \nu \nabla^{s} \delta \boldsymbol{v} \cdot \Delta p \mathbf{1} d V \\
\Delta \delta_{\sigma p} \mathcal{J}= & \frac{1}{\rho \nu} \int_{\Omega} \delta \boldsymbol{\sigma} \cdot \Delta p \mathbf{1} d V .
\end{aligned}
$$

The quantities $\Delta \delta_{\sigma v} \mathcal{J}, \Delta \delta_{p v} \mathcal{J}$ and $\Delta \delta_{p \sigma} \mathcal{J}$ could be obtained by interchanging $\delta$ with $\Delta$. Alternatively, the Newton tangent can also be computed by a standard difference quotient procedure. The discretization of the domain is realized by triangles equipped with different conforming interpolation schemes for the unknown variables. For the velocities and pressure we use the finite element spaces

$$
\boldsymbol{V}_{k}^{h}=\left\{\boldsymbol{v} \in H^{1}(\hat{\Omega})^{2}:\left.\boldsymbol{v}\right|_{\hat{\Omega}} \in P_{k}(\hat{\Omega})^{2} \forall \hat{\Omega}\right\} \subset \boldsymbol{V}
$$

and

$$
\boldsymbol{Q}_{l}^{h}=\left\{p \in L_{2}(\hat{\Omega})^{2}:\left.p\right|_{\hat{\Omega}} \in P_{l}(\hat{\Omega})^{2} \forall \hat{\Omega}\right\} \subseteq \boldsymbol{Q},
$$

where the indices $k$ and $l$ denote the polynomial order of the standard Lagrange polynomials $P_{k}(\hat{\Omega})^{2}$ and $P_{l}(\hat{\Omega})^{2}$, and $\hat{\Omega}$ is the disretized version of the domain $\Omega$. For the stresses a Raviart-Thomas finite element space, see e.g. [41] or [42], is applied in order to complete the $R T_{m} P_{k} P_{l}$ mixed finite element

$$
\boldsymbol{X}_{m}^{h}=\left\{\boldsymbol{\sigma} \in H(\operatorname{div}, \hat{\Omega})^{2}:\left.\boldsymbol{\sigma}\right|_{\hat{\Omega}} \in R T_{m}(\hat{\Omega})^{2} \forall \hat{\Omega}\right\} \subset \boldsymbol{X} .
$$

Again the index $m$ denotes the polynomial order of the vector-valued Raviart-Thomas functions $R T_{m}$, which interpolate each row of the stress tensor. These functions are related to the edges of the triangles and their normal components are continuous between two elements. In this work we use the interpolation combination $R T_{1} P_{3} P_{1}$, which seems to be a sufficient choice. For details about the implementation and other interpolation combinations the reader is referred to [40]. Finally, we obtain the system of equations for a typically element

$$
\left[\begin{array}{ccc}
\boldsymbol{k}_{v v}^{e} & \boldsymbol{k}_{v \sigma}^{e} & \boldsymbol{k}_{v p}^{e} \\
\boldsymbol{k}_{\sigma v}^{e} & \boldsymbol{k}_{\sigma \sigma}^{e} & \boldsymbol{k}_{\sigma p}^{e} \\
\boldsymbol{k}_{p v}^{e} & \boldsymbol{k}_{p \sigma}^{e} & \boldsymbol{k}_{p p}^{e}
\end{array}\right]\left[\begin{array}{c}
\Delta \boldsymbol{v}^{e} \\
\Delta \boldsymbol{\sigma}^{e} \\
\Delta \boldsymbol{p}^{e}
\end{array}\right]=\left[\begin{array}{c}
\boldsymbol{r}_{v}^{e} \\
\boldsymbol{r}_{\sigma}^{e} \\
\boldsymbol{r}_{p}^{e}
\end{array}\right]
$$

where the entries of $\boldsymbol{r}^{e}$ and $\boldsymbol{k}^{e}$ result from 12 and $(13)$. 


\subsection{LSFEM with data (LSFEM+)}

The Navier-Stokes equations were formulated as a minimization problem in the previous section. This form suggests an simple adjustment to incorperate experimental data. Consider data $\boldsymbol{d} \in \mathbb{R}^{M}$ which is approximated by $H w$ where $H: V \rightarrow \mathbb{R}^{M}$ is the observation operator. The implication is that $\boldsymbol{d} \approx H w$. Then a possible LSFEM functional $\mathcal{J}(w)$ becomes

$$
\mathcal{J}^{+}(w):=\mathcal{J}(w)+\frac{1}{2} \sum_{i=1}^{M} \omega_{i}\left(d_{i}-H_{i} w\right)^{2},
$$

i.e. differences between data and state are penalized. Here $\omega_{i}$ are per-measurement weights.

This is a useful modification in the following circumstances: a fluid-dynamics experiment is performed, in which some (limited) quantities are measured. E.g. velocity $\boldsymbol{v}$ at a limited number of spatial locations in $\Omega$; pressure $p$ at a number of pressure taps on the surface $\Gamma$; or a force in the form of an integral of $p$ over $\Gamma$. These measurements may not contain the Quantity-of-Interest (QoI) of the experimentor because it is inaccessable or unmeasurable; or they may be noisy or of low-resolution. It is desired to estimate the QoI from the measured data, using knowledge of the equations of motion of the fluid (in the form of a PDE solver). However, conditions in experiments are never precisely known: e.g. boundary conditions are uncertain, and therefore can not be set in the solver.

By augmenting with data, the simulation becomes a better reproduction of the experiment from which the data originated - and the QoI can be estimated based on a composite of knowledge derived from measurement and simulation. The solver can be thought of as performing PDE-based regression in the state-space.

In Section 3 we will formulate more precisely the problem that is being solved, and justify the choice of the functional 18 in terms of Bayesian statistics. A close connection to standard data-assimilation techniques will be demonstrated.

\section{Bayesian derivation of LSFEM data-assimilation}

In the following we formulate the data-assimilation problem, and provide a Bayesian resolution. The maximum a posteriori (MAP) estimate of the posterior is shown to be exactly the solution of the augmented LSFEM problem of the previous section. The close connection to the Kalman filter (and equivalently 3DVAR with a suitable background field) is demonstrated. This development is made in detail for the linear case. The idea could be extended to the nonlinear case - for an empirical evaluation the approach for N-S see Section 4. In conclusion, augmented LSFEM is shown to produce a solution that is identical to standard data-assimilation approaches - but for a substancially reduced computational cost.

\subsection{Problem statement and data model}

Problem 1 (Data assimilation). Let $w^{*} \in V$ be the unknown ground truth. Knowledge of $w^{*}$ comes from two sources: 
1. Finite, noisy measurements $\boldsymbol{d} \in \mathbb{R}^{M}$, obtained from $w^{*}$ using the known observation operator $H: V \rightarrow \mathbb{R}^{M}$, i.e.

$$
\boldsymbol{d} \simeq H w^{*} .
$$

2. Approximate knowledge of the governing equations and boundary-conditions, i.e.

$$
L w^{*}-f \simeq 0 \text { on } \Omega, \quad B w^{*}-g \simeq 0 \text { on } \Gamma .
$$

Estimate $w^{*}$.

To characterize the inexact equalities in $(19)-(20)$, we introduce statistical models. In (19) assume that observations are corrupted by unbiased Gaussian measument noise with known symmetric positive-definite covariance matrix $\boldsymbol{R} \in \mathbb{R}^{M \times M}$, i.e.

$$
\boldsymbol{d}=H w^{*}+\varepsilon, \quad \varepsilon \sim \mathcal{N}(\mathbf{0}, \boldsymbol{R}) .
$$

The assumption of unbiased noise is reasonable if all known sources of bias are corrected for. (Approximately) known covariance is a reasonable assumption if experimental noise has been estimated concurrent with the measurements. Both these are features of modern experimental methodologies. No stochastic term for "model inadequacy" in $H$ [3] is included, under the assumption that $H$ is a relatively simple operator that merely extracts necessary quantities from the state $w^{*}$, and does not contain any physical modelling or discretization. This is the case for many observation operators of practical interest, including the "point velocity observations" used in the following section.

\subsection{Model inadequacy in the governing equations}

In contrast 20 contains both physical modelling, discretization, and potentially unknown/uncertain equations-of-state and boundary-conditions. All these are reasons for the ground truth to not exactly satisfy the (discretized) governing equations (2). In variational form consider a modified version of $(2)$ which the ground-truth does satisfy:

$$
Q\left(w^{*}, v\right)=l(v)+\delta(v) \quad \forall v \in V,
$$

where the new functional $\delta: V \rightarrow \mathbb{R}$, represents the discrepancy, and could be defined by $\delta(v):=l(v)-Q\left(w^{*}, v\right)$ if the ground truth $w^{*}$ were known. In practice $\delta(v)$ must be modelled, and following [44] we treat it as a Gaussian process

$$
\delta(v) \sim \mathcal{G P}(0, k(w, v)),
$$

with zero mean and covariance operator $k: V \times V \rightarrow \mathbb{R}$. The resulting equation for $w$ is a stochastic PDE:

$$
Q(w, v)=l(v)+\delta(v) \quad \forall v \in V .
$$

In [44] the authors consider a parameterized family of covariance operators $k(\cdot, \cdot)$ :

$$
k(w, v ; \boldsymbol{\theta}):=\theta_{1}(w, v)_{\Omega}+\theta_{2}(\nabla w, \nabla v)_{\Omega},
$$

representing the $H^{1}(\Omega)$ scalar product. Here we propose a covariance operator as a scalar product informed by the governing PDE:

$$
k(w, v ; \boldsymbol{\theta}):=\theta_{1}(L w, L v)_{\Omega}+\theta_{2}(B w, B v)_{\Gamma},
$$


which is symmetric positive-definite

$$
k(w, v)=k(v, w), \quad k(v, v)>0 \text { for } v \neq 0, \quad k(v, v)=0 \text { for } v=0
$$

by construction, provided $L$ is bounded below. This condition is in any case a prerequisite for the stability and accuracy of LSFEM - in particular it is true for the Navier-Stokes operator of the previous section.

\subsection{Calibration}

It remains to compute the posterior $w \mid \boldsymbol{d}$ under the statistical model (21) and prior (23) - i.e. the updated estimate of the full state. First it is necessary to discretize to obtain finite-dimensional equations, with basis $\left(\phi_{1}, \ldots, \phi_{N}\right)$ :

$$
w \simeq w_{h}:=\sum_{i=1}^{N} \boldsymbol{w}_{i} \phi_{i},
$$

where discrete quantities are represented by bold type. Given this 211 becomes

$$
\boldsymbol{d}=\boldsymbol{H} \boldsymbol{w}+\boldsymbol{\varepsilon}, \quad \boldsymbol{H}_{i j}:=\left(H \boldsymbol{\phi}_{j}\right)_{i},
$$

and the prior on the disretized discrepancy $\boldsymbol{\delta}$ is

$$
\boldsymbol{\delta}_{0} \sim \mathcal{N}(\mathbf{0}, \boldsymbol{\Sigma}), \quad \boldsymbol{\Sigma}_{i j}:=k\left(\phi_{i}, \phi_{j}\right)
$$

Furthermore, from (24)

$$
\boldsymbol{Q w}=\boldsymbol{l}+\boldsymbol{\delta}, \quad \boldsymbol{Q}_{i j}:=Q\left(\phi_{i}, \phi_{j}\right), \boldsymbol{l}_{j}:=l\left(\phi_{j}\right) .
$$

Note that, if the weights in the definitions of $Q(\cdot, \cdot)$ and $k(\cdot, \cdot)$ are equal, then $\boldsymbol{Q} \equiv \boldsymbol{\Sigma}$, which is a direct consequence of the particular choice of prior covariance for $\boldsymbol{\delta}$. This observation, together with 26) implies a prior on $\boldsymbol{w}$ :

$$
\boldsymbol{w}_{0} \sim \mathcal{N}\left(\boldsymbol{Q}^{-1} \boldsymbol{l}, \boldsymbol{Q}^{-1} \boldsymbol{\Sigma} \boldsymbol{Q}^{-1}\right)=\mathcal{N}\left(\boldsymbol{w}^{\circ}, \boldsymbol{Q}^{-1}\right),
$$

where $\boldsymbol{Q} \boldsymbol{w}^{\circ}=\boldsymbol{l}$ defines $\boldsymbol{w}^{\circ}$ as the solution of the original LSFEM discretization (without a data term). Having eliminated $\boldsymbol{\delta}$ we can apply Bayes' theorem to $\boldsymbol{w}$ :

$$
\rho(\boldsymbol{w} \mid \boldsymbol{d}) \propto \rho(\boldsymbol{d} \mid \boldsymbol{w}) \cdot \rho_{0}(\boldsymbol{w}) .
$$

Since all stochastic terms are Gaussian the posterior probability density function (pdf) is

$$
\begin{aligned}
\rho(\boldsymbol{w} \mid \boldsymbol{d}) & =\rho_{\varepsilon}(\boldsymbol{\varepsilon}) \cdot \rho_{0}(\boldsymbol{w}) \\
& =\exp \left[-\frac{1}{2} \varepsilon^{T} \boldsymbol{R}^{-1} \boldsymbol{\varepsilon}-\frac{1}{2}\left(\boldsymbol{w}-\boldsymbol{w}^{\circ}\right)^{T} \boldsymbol{Q}\left(\boldsymbol{w}-\boldsymbol{w}^{\circ}\right)\right] .
\end{aligned}
$$

The MAP estimate of $\boldsymbol{w}$ is therefore the solution of the optimization problem

$$
\boldsymbol{w}^{+}:=\operatorname{argmin}_{\boldsymbol{w}}\left[\frac{1}{2}(\boldsymbol{d}-\boldsymbol{H} \boldsymbol{w})^{T} \boldsymbol{R}^{-1}(\boldsymbol{d}-\boldsymbol{H} \boldsymbol{w})+\frac{1}{2}\left(\boldsymbol{w}-\boldsymbol{w}^{\circ}\right)^{T} \boldsymbol{Q}\left(\boldsymbol{w}-\boldsymbol{w}^{\circ}\right)\right],
$$


and the relation to LSFEM is immediately obvious. In particular, setting 1st-variations of the cost-function to zero gives

$$
\boldsymbol{w}^{T} \boldsymbol{Q} \delta \boldsymbol{w}+\boldsymbol{w}^{T} \boldsymbol{H}^{T} \boldsymbol{R}^{-1} \boldsymbol{H} \delta \boldsymbol{w}=\boldsymbol{d}^{T} \boldsymbol{H}^{T} \boldsymbol{R}^{-1} \delta \boldsymbol{w}+\boldsymbol{l}^{T} \delta \boldsymbol{w}, \quad \forall \delta \boldsymbol{w} \in \boldsymbol{V}
$$

which is exactly the discrete version of the LSFEM problem with data-term from 18): Find $w \in V$ such that

$$
\underbrace{(L w, L \delta w)_{\Omega}+(B w, B \delta w)_{\Gamma}}_{Q(w, \delta w)}+\underbrace{(H w, H \delta w)_{R}}_{T(w, \delta w)}=\underbrace{(f, L \delta w)_{\Omega}+(g, B \delta w)_{\Gamma}}_{l(\delta w)}+\underbrace{(\boldsymbol{d}, H \delta w)_{R}}_{t(\delta w)} \quad \forall \delta w \in V .
$$

provided that $[\boldsymbol{R}]_{i i}=\omega_{i}$ and $[\boldsymbol{R}]_{i j}=0$ for $i \neq j$. In the above, the inner-product for the data is defined as

$$
(\boldsymbol{x}, \boldsymbol{y})_{R}:=\boldsymbol{x}^{T} \boldsymbol{R}^{-1} \boldsymbol{y}
$$

Thus the Bayesian framework - with appropriate statistical modelling - leads us to the LSFEM+.

Note that the connection between LSFEM+ and the statistical data-assimilation problem relies upon the particular choice of an additive discrepency $\delta$ in $(22)$, with the specific covariance defined by (25). If an alternative covariance structure for $\boldsymbol{\delta}$ is chosen, e.g. from [44, LSFEM does not provide a solution of the corresponding inverse problem.

\subsection{Connection to the Kalman filter}

We would like to describe the the solution of $(28)\left(\right.$ denoted $\left.w^{+}\right)$, in terms of the solution to the same equation without data (2) $\left(w^{\circ}\right)$. The goal is to show the result is identical to the application of a Kalman filter.

Starting from (27), cancelling $\boldsymbol{l}$ using $\boldsymbol{Q} \boldsymbol{w}^{\circ}=\boldsymbol{l}$, and rearranging gives

$$
\begin{aligned}
\boldsymbol{w}^{+} & =\boldsymbol{w}^{\circ}+\left(\boldsymbol{Q}+\boldsymbol{H}^{T} \boldsymbol{R}^{-1} \boldsymbol{H}\right)^{-1}\left(\boldsymbol{H}^{T} \boldsymbol{R}^{-1} \boldsymbol{d}-\boldsymbol{H}^{T} \boldsymbol{R}^{-1} \boldsymbol{H} \boldsymbol{u}^{\circ}\right) \\
& =\boldsymbol{w}^{\circ}+\left[\left(\boldsymbol{Q}+\boldsymbol{H}^{T} \boldsymbol{R}^{-1} \boldsymbol{H}\right)^{-1} \boldsymbol{H}^{T} \boldsymbol{R}^{-1}\right]\left(\boldsymbol{d}-\boldsymbol{H} \boldsymbol{u}^{\circ}\right) \\
& =\boldsymbol{w}^{\circ}+\underbrace{\boldsymbol{Q}^{-1} \boldsymbol{H}^{T}\left(\boldsymbol{H} \boldsymbol{Q}^{-1} \boldsymbol{H}^{T}+\boldsymbol{R}\right)^{-1}}_{\boldsymbol{K}}\left(\boldsymbol{d}-\boldsymbol{H} \boldsymbol{u}^{\circ}\right),
\end{aligned}
$$

where the final equality is obtained using a modified version of the matrix identity

$$
\left(\boldsymbol{Q}+\boldsymbol{H}^{T} \boldsymbol{R}^{-1} \boldsymbol{H}\right)^{-1} \boldsymbol{H}^{T} \boldsymbol{R}^{-1} \equiv \boldsymbol{Q}^{-1} \boldsymbol{H}^{T}\left(\boldsymbol{H} \boldsymbol{Q}^{-1} \boldsymbol{H}^{T}+\boldsymbol{R}\right)^{-1} .
$$

The matrix $\boldsymbol{K}$ is the well-known optimal Kalman gain, under the statistical model 21, and the background covariance model $\boldsymbol{w} \sim \mathcal{N}\left(\boldsymbol{w}^{\circ}, \boldsymbol{Q}^{-1}\right)$. In fact $\boldsymbol{w}^{+}$is the MAP estimate of $\boldsymbol{w} \mid \boldsymbol{d}$ with distribution

$$
\boldsymbol{w} \mid \boldsymbol{d} \sim \mathcal{N}\left(\boldsymbol{w}^{+},(\boldsymbol{I}-\boldsymbol{K} \boldsymbol{H}) \boldsymbol{Q}^{-1}\right) .
$$

This result thereby also provides an expression for the posterior covariance of the full state $\boldsymbol{w}$. 


\section{Application: Laminar Flow over a Backward-Facing Step}

The phenomena of flow separation and subsequent re-attachment are important in many engineering situations. For example in pipe flow separation is undesirable and leads to reduction in flow rate for a given pressure-drop, requiring additional pumps or fans. In other circumstances separation may be desirable, to promote mixing or flame stabilization in combustion. However the presence and location of separation on curved surfaces, and the location of re-attachment in general, are difficult to predict, and are sensitive to small uncertainties in inflow conditions and fluid properties.

We aim to show that using down-stream measurements and simulation it is possible to accurately determine the location of the re-attachment point in the presence of uncertain boundary-conditions. We consider a backward-facing step in laminar flow for which accurate experimental velocity profiles are available, Denham and Patrick 45, 46]. This experiment is especially appropriate for this study, as it is supposed to model an ideal situation in which the inflow-boundary is a fully-developed channel-flow (with a parallel, parabolic velocity-profile), but due to experimental limitations the inflow is far from fully-developed. In [45] the actual inflow profile is also measured, and this will be used as a reference.

The setup and material parameters of the backward-facing step can be found in Figure 1. The Reynolds number is $\mathrm{Re}=191$ based on the step height and inflow peak velocity [45. The circulation length is roughly $x_{l}=12.7$. We prescribe no-slip BCs on the lower- and upper walls and a zero pressure BC on the right-hand outflow. On the left-hand side we consider a variety of BCs representing different states of knowledge of the inflow condition.

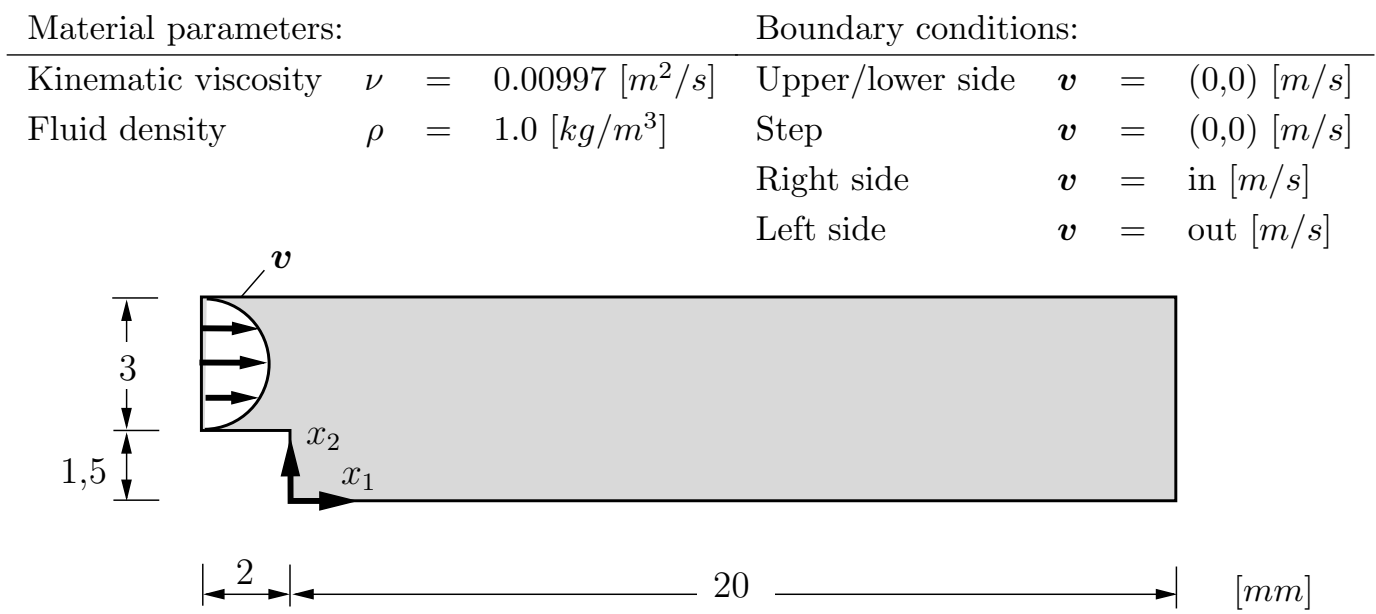

Figure 1: Material parameters and geometry of the backward-facing step.

Due to the low Reynolds-number the flow is laminar, and therefore no turbulence modelling is required, eliminating a potential source of modelling inadequacy which would have been difficult to estimate and control [43. Errors in our solutions are therefore all due to discretization, and due to the imprecisely known inflow boundary-condition. We 
examine the effect of adding data on these two error sources. We numerically investigate: (i) improving the accuracy of the predictions by adding data in Section 4.3 . (ii) solving the inverse problem in which the inflow BC is completely unknown and unspecified 4.4.1. and (iii) exploiting partial, uncertain knowledge of the inflow to define the flow 4.4 .2

The discretization uses cubic $R T_{1} P_{3} P_{1}$ elements, on two triangular meshes: a coarse mesh with 528 nodes and a fine mesh of 8770 nodes. Results will be compared to experimental velocity profile measurements at four downstream locations: $x=3, x=6$, $x=9, x=12$, and the inflow plane $x=-2$.

\subsection{Data Acquisition and Processing}

Denham and Patrick [45] used a highly accurate non-invasive laser Döpplar anemometry system to measure the $x$-component of the flow velocity vector. They report smooth, wellresolved velocity profiles and give error estimates. The anemometry itself is described as being accurate to $0.003 \mathrm{~mm} / \mathrm{s}+2 \%$ of its value. In addition we obtained the data in fine, by electronically scanning the hand-plotted graphs of [45] (the only available source), see Figure 2. An additional inaccuracy arises in this process which we estimated to be $5 \%$. These values inform our choice of $\boldsymbol{R}$. Of other experimental errors, the authors consider $3 \mathrm{~d}$ effects to be negligible, but report the non-fully developed inflow, and a time-varying flow-rate of $0.5 \%$ as potentially significant error sources. These errors will be accounted for via the uncertain inflow condition.

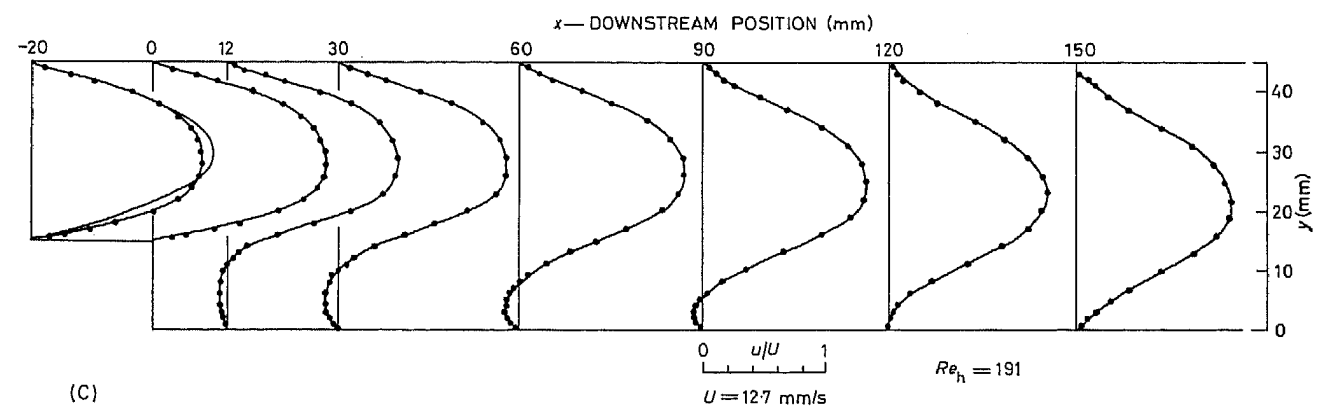

Figure 2: Reproduction of Figure 5(c) from Denham and Patrick, 1974 [45]. Velocity profiles for a backward-facing step at $\operatorname{Re}=191$.

For our implementation it is convenient to have measurement data (and error) available at the nodes of the finite-element mesh. For this purpose we regress the digitized velocity profile points with a Kriging surface 47. Kriging is a stochastic response surface methodology, and as such can account for noise in data, and predict uncertainties at reconstruction points [48, 49. We use the capability to construct $\boldsymbol{R}$ at the FE-mesh nodes. See Figure 3, for an example reconstruction at $x=3.0$.

\subsection{Error Study for the Simulation without Data}

Before looking at data assimilation we investigate the performance of the numerical simulation in the absence of experimental data in order to study sources of error. Critically 


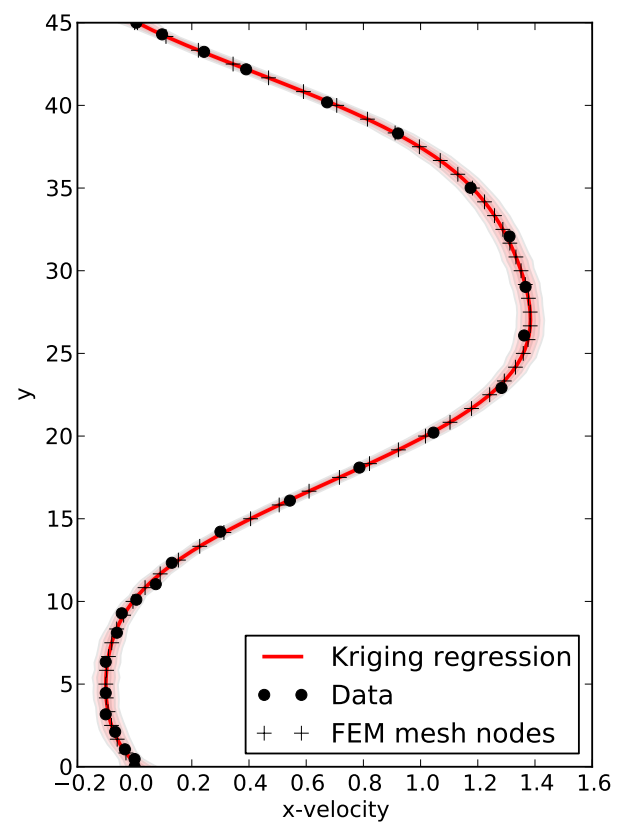

Figure 3: Horizontal velocity component at $\mathrm{Re}=191, x=3.0$. Original measurements, Kriging response mean and $3 \sigma$ with assumption of $5 \%$ noise in measurements, and values interpolated at computational nodes.

here we examine error with respect to experimental data - not with respect to a finegrid simulation. Therefore not only are discretization errors visible, but also modelling inadequacy, and uncertainties in the boundary conditions and material properties [43]. We demonstrate that for a reasonably fine mesh, the inflow BC uncertainty dominates the discretization error, and therefore is more important for correctly reproducing the experiment, and presumably for prediction.

Firstly we consider the case of a parabolic velocity profile at the inflow boundary (Poiseuille flow):

$$
\boldsymbol{v}=\left(4 v_{\max } y(h-y) / h^{2}, 0\right)^{T}
$$

where $h \in[0,3]$ is the height of the inflow and the assumed peak inflow velocity is $v_{\max }=1.81$. In the absence of measurement data, this is the simulation practitioner's best-guess of the inflow condition, representing fully-developed laminar pipe-flow, see Section 4.1 .

Figure 4 shows results for the discretizations of 528 and 8770 nodes as well as the reference solution of [45] at four $x$-locations. It can be seen that although the fine grid solution gives a substantial accuracy improvement over the coarse grid, neither approaches the experimental reference. In particular the flow reattachment location in the experiment is at $x_{l}=12.7$, while on the fine grid it is at $x_{l}=14.5$. Further refinement of the mesh shows no substantial change. Given that modelling inadequacy 
is also expected to be small, we conclude that the largest part of the remaining error is due to imprecise reproduction of the experimental conditions.

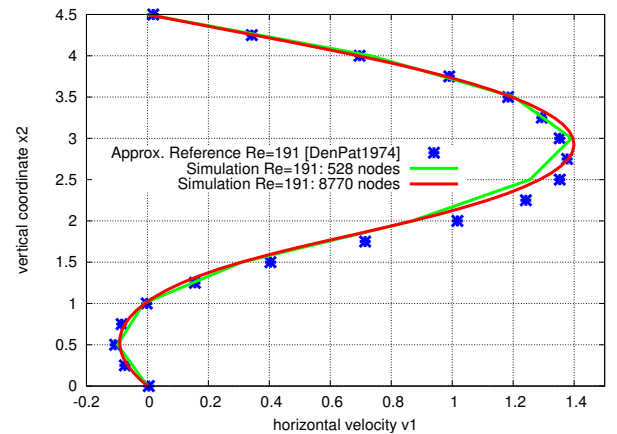

(a) $x=3$

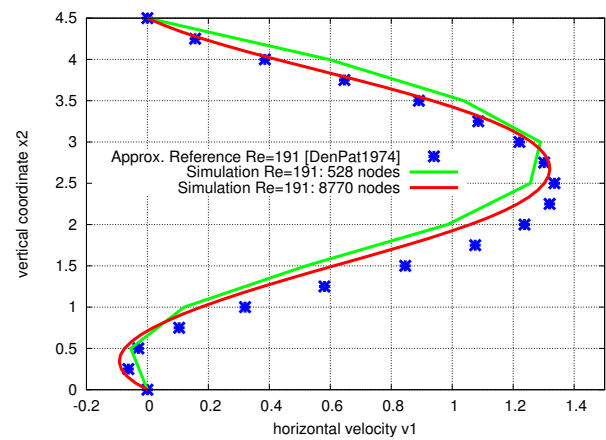

(c) $x=9$

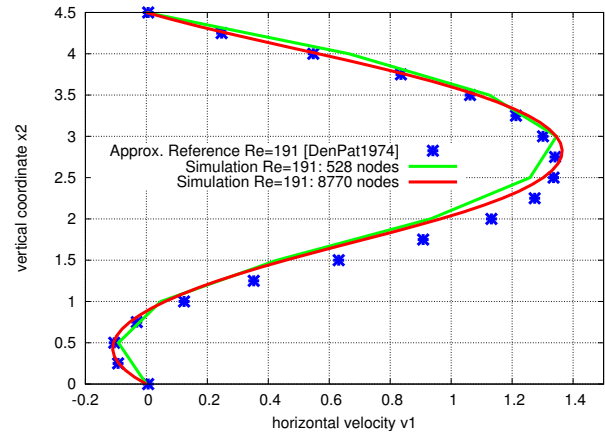

(b) $x=6$

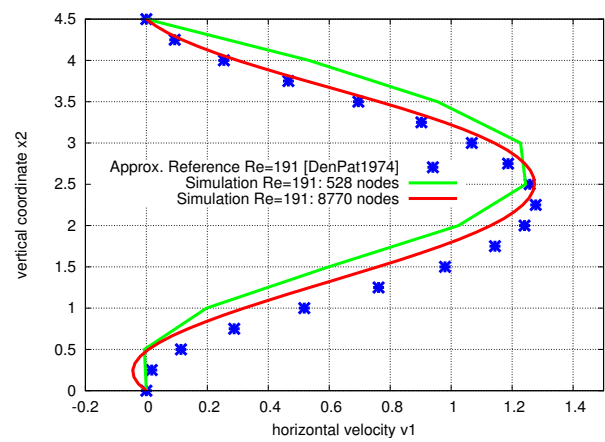

(d) $x=12$

Figure 4: Horizontal velocity profiles. Parabolic inflow condition on a coarse and fine mesh.

From Figure 2 we see that actual the inflow profile measured in the experiment significantly deviates from a parabola. Therefore in the following computations the inflow BC is changed to the measured velocities from the experiment, interpolated with Kriging. The previous computations are repeated with the new BC, and results depicted in Figure 5. It can be seen that for the fine grid the largest part of the discrepancy is removed. The remaining small differences can be attributed to uncertainties in the unmeasured vertical component of the inflow velocity. The recirculation length is now predicted as $x_{l}=13.3$, also significantly improved. The coarse grid solution remains poor — discretization error clearly dominates here.

This demonstrates one case in which the leading-order error with respect to reality can be discretization error or BC uncertainty, depending on the mesh. We aim now to estimate and reduce both these types of error with the injection of measurement data downstream of the inflow. 


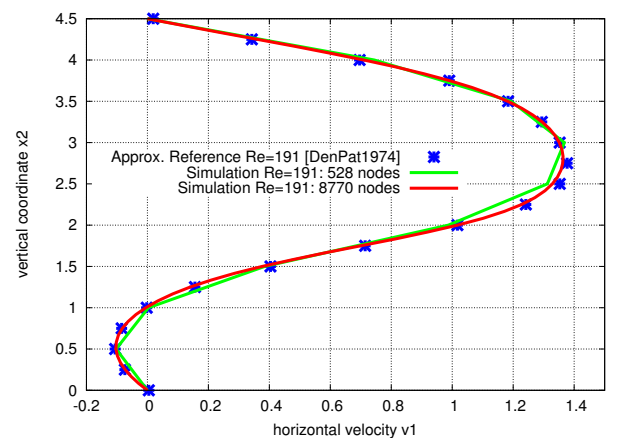

(a) $x=3$

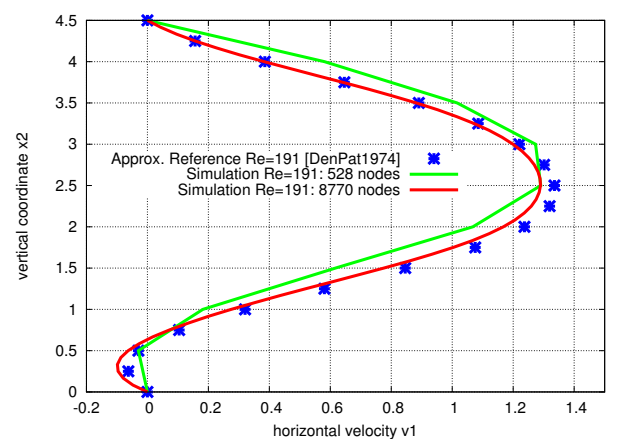

(c) $x=9$

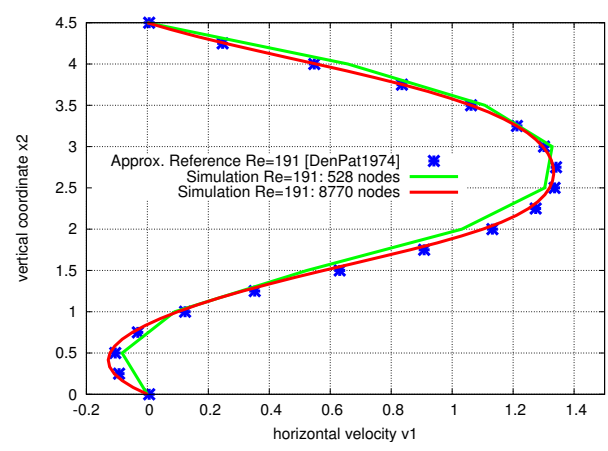

(b) $x=6$

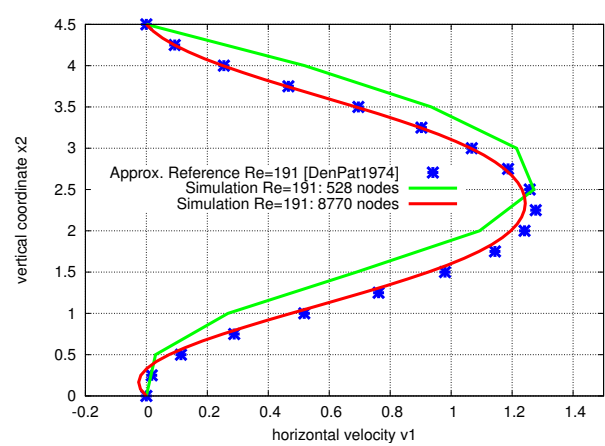

(d) $x=12$

Figure 5: Horizontal velocity profiles. Inflow from experiment, coarse and fine grids.

\subsection{Correcting Discretization Error with Experimental Data}

For the coarse grid solution in Figure 5 we observed a discretization error which appeared to dominate the total error with respect to reality. We examine the ability of data injection downstream to reduce this large discretization error. Therefore, we study the same cases as previously, with the inflow BC specified from the experiment.

The experimental data is included in the form of a velocity profile at either $x_{d}=9$ or $x_{d}=15$, see Figure 6. The former reversed flow near the wall; at the latter station the flow in the experiment is already uni-directional. Figure 7 shows the results for the coarse mesh without and with experimental data as well as the experimental reference solution of [45] at $x=3, x=6, x=9$ and $x=12$.

It can be seen that at all locations a substantial reduction in the error is achieved, not only at the location of the added data, but in the entire domain. The improvement is most noticeable for $x_{d}=9,12$, where the original discretization error is largest (due to mass loss and accumulation of errors from upstream), and where the data has the most influence. In Figure 7(c) for $x=9$, and data at the same location $x_{d}=9$, we can see the local influence of the data term. The solution is prescribed mainly by the data at this point, and follows it closely. Note that due to the extremely coarse grid cells, the recirculation region at this location is almost not resolved in the data set. This limits the 
improvement of the approximation of this small feature. At $x_{d}=15$ the data contains no recirculation region, yet an improvement in the location of the maximum velocity at all $x$-stations is still observed.

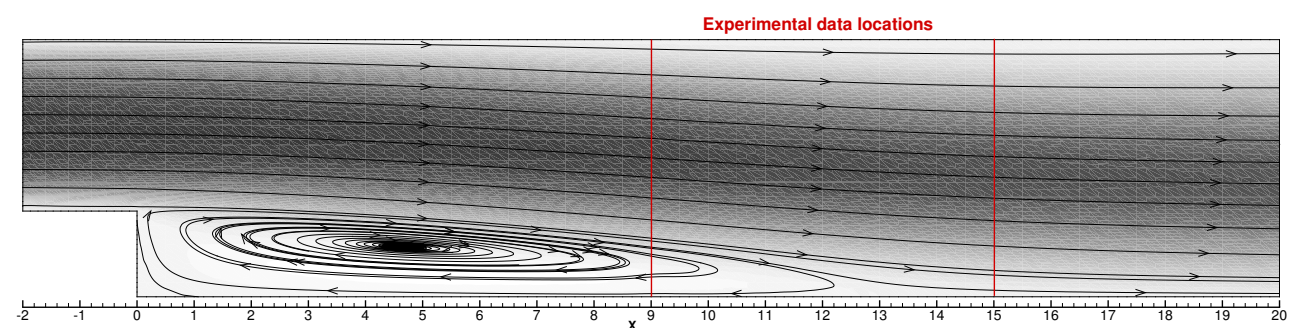

Figure 6: Streamlines and contours of horizontal-flow velocity for the simulation on the fine mesh, with experimental inflow $\mathrm{BC}$, and experimental data inserted at $x=9$. Alternative data location at $x=15$ also marked.

For the fine grid in Figure 5 there is also a defect, though in this case it is not primarily due to discretization error. Nonetheless we apply the same procedure as for the coarse grid, with data introduced at the same locations in the hope that this unidentified error will be reduced, see Figure 8 . Since the fine grid has more nodes in the $y$-direction, more data is used, in particular the reversed-flow region at $x_{d}=9$ is resolved. Again we see reduction in error across the full domain for data introduced at both $x_{d}=9$ and $x_{d}=15$. This time, not only the location and strength of the peak is the benefactor, but also the reproduction of the circulation region.

\subsection{Correcting Unknown and Uncertain BCs with Experimental Data}

Reducing discretization error can be achieved most readily with mesh refinement. However in Section 4.2 it was seen that for a reasonable mesh resolution, error in the inflow $\mathrm{BC}$ dominated the total error balance. Such errors can not be reduced without reference to the experiment. Consider the situation in which the inflow conditions to the computational domain are unmeasured. This frequently occurs in experiments in fluids due to issues of measurement device access, for example, optical access in PIV.

In order to imitate this situation we consider two possible states of knowledge regarding the horizontal inflow boundary condition. Firstly we assume absolutely no information regarding the inflow velocity is available, secondly we assume that the inflow is known to be roughly parabolic (as follows from basic flow theory). The experimental data is included at $x=9$, see Section 4.3 Furthermore, we assume zero vertical velocity at the inflow in both cases. The setup and material parameters of the backward facing step at Reynolds number of $R e=191$ are the same as in the previous section. 


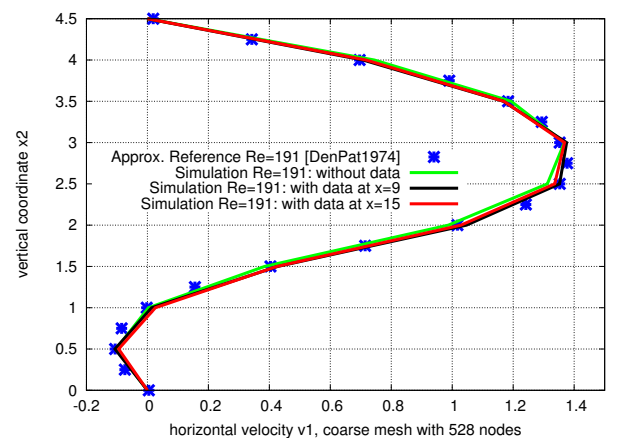

(a) $x=3$

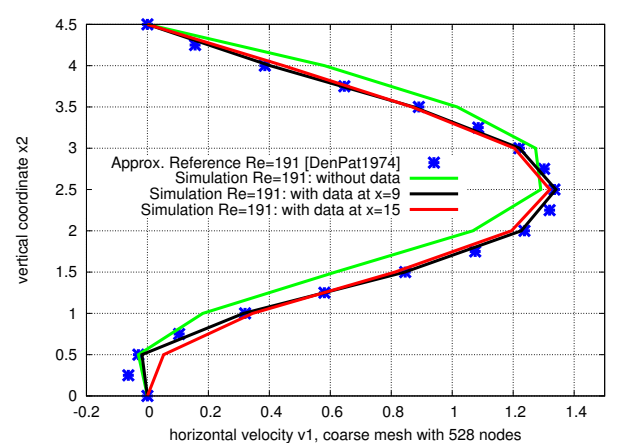

(c) $x=9$

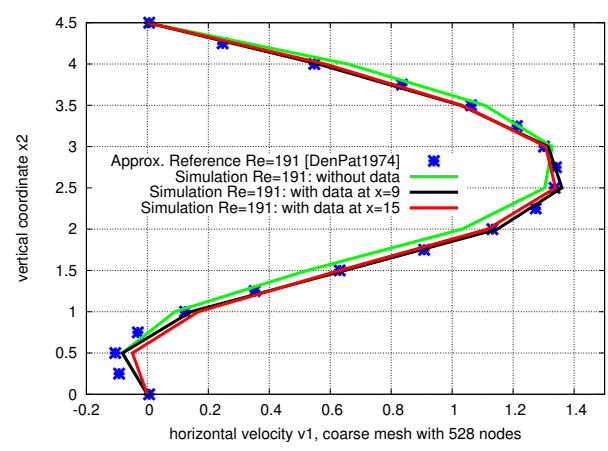

(b) $x=6$

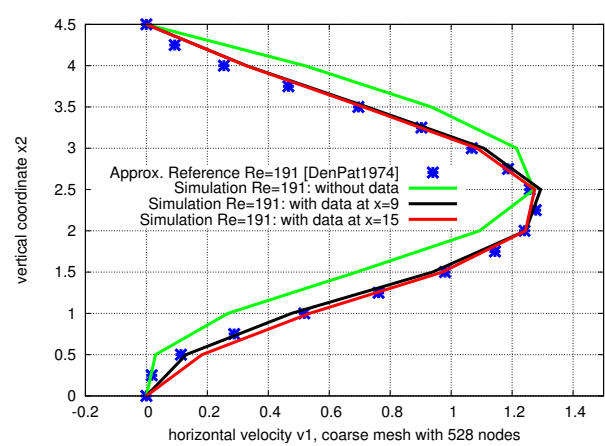

(d) $x=12$

Figure 7: Horizontal velocity profiles. Coarse grid, experimental data at $x=9$ or $x=15$.

\subsubsection{Simulation with fully unknown inflow conditions}

Here we consider the situation in which we assume no information about the inflow condition. We simply omit from the discretization all horizontal inflow BCs (weak and strong). This type of boundary value problem is a classic inverse problem. The results for the discretizations of 528 and 8770 nodes as well as the reference solution from the experiment at the inflow at $x=3, x=6$ and $x=12$ are depicted in Figure 9. We leave the results at $x=9$ out because they are identical to the reference solution as in Section 4.3 .

From Figure 9(a) it is clear that the procedure reproduces a physically reasonable inflow profile, that matches roughly the reference profile from the experiment. Most of the error is related to an overestimation of the maximum velocity and the mass flow. Such an error could be due to the discretization losing some mass in the channel. When solving the inverse problem the method therefore injects extra mass to account for this loss. The closer we get to $x=9$ the closer the results become to the experimental reference solution. It can be seen that our proposed approach is capable of solving problems with unknown BCs in a quite satisfying manner. 


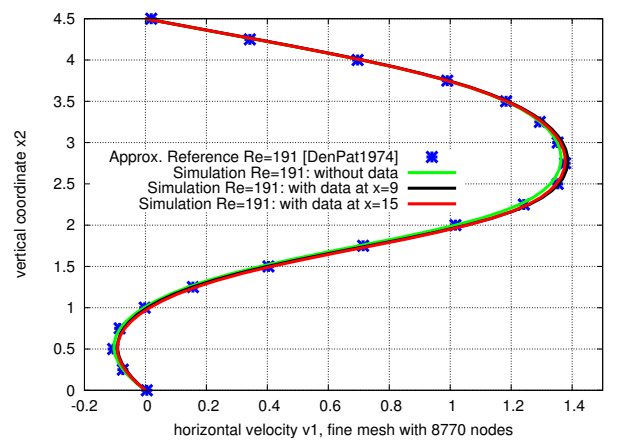

(a) $x=3$

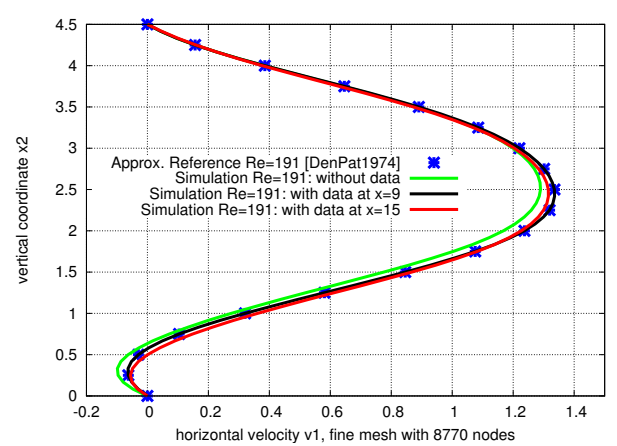

(c) $x=9$

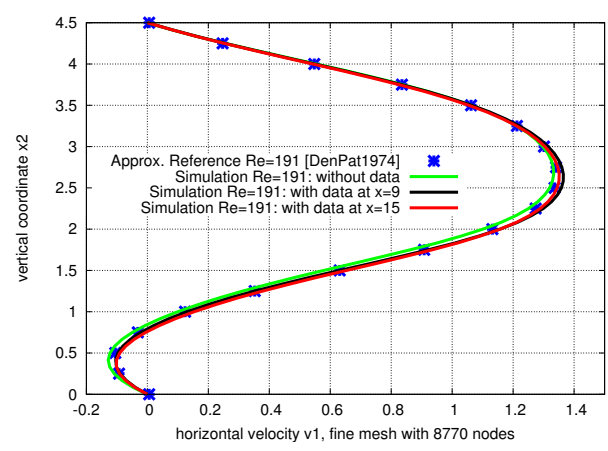

(b) $x=6$

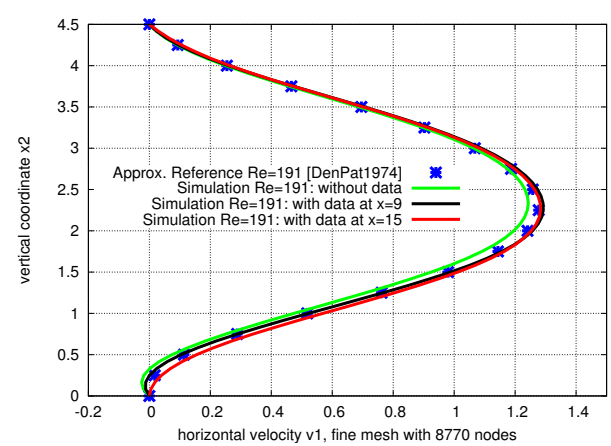

(d) $x=12$

Figure 8: Horizontal velocity profiles. Fine grid, experimental data at $x=9$ or $x=15$.

\subsubsection{Simulation with weak parabola inflow conditions}

Now we try to improve on the results of the previous section by incorporating some inexact knowledge of the horizontal inflow velocity - namely that it is roughly parabolic with an estimated maximum velocity. We employ the parabola from Section 4.2 but in order to accommodate the uncertainty in this assumption we include the parabolic $\mathrm{BC}$ in weak form with a coefficient of $\omega=0.01$. The implementation of weak boundary conditions is realized in similar form to the assimilation data term. As in the previous section we omit the results at $x=9$ and consider the results at the inflow $(x=-2)$, and at $x=3,6,12$ for the fine and coarse discretizations. In Figure 10 the results for the horizontal velocity profiles with weak parabolic inflow boundary conditions are depicted.

Looking first at Figure $10(\mathrm{a})$, we see an improvement in the quality of the velocity profile on the fine grid at the inflow. Most noticeably, the bump present at $y=4$ in Figure 9(a) is removed. However also on the lower side of the profile, the experimental match is slightly better, and the overall mass flow is substantially corrected. Downstream the effect is less pronounced, and very little difference with respect to the no-BC case is visible. Overall we see that using approximate information at the inflow in the form of a weak BC, gives an improved solution compared to using no information there. 


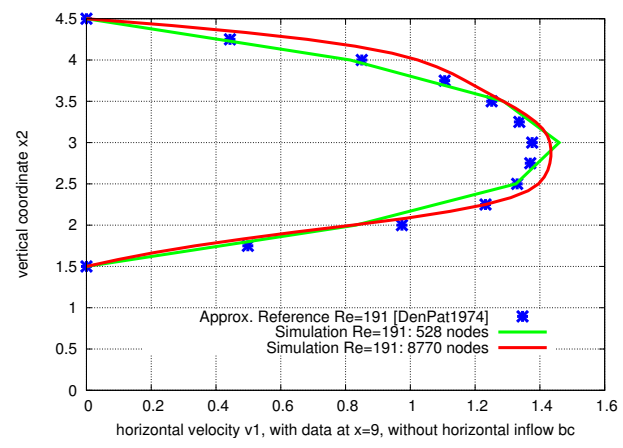

(a) $x=-2$

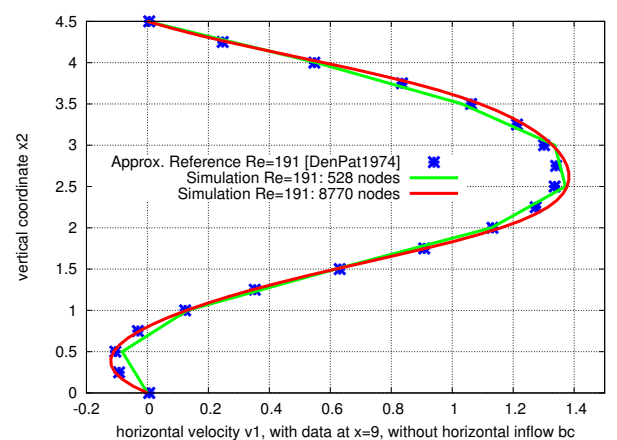

(c) $x=6$

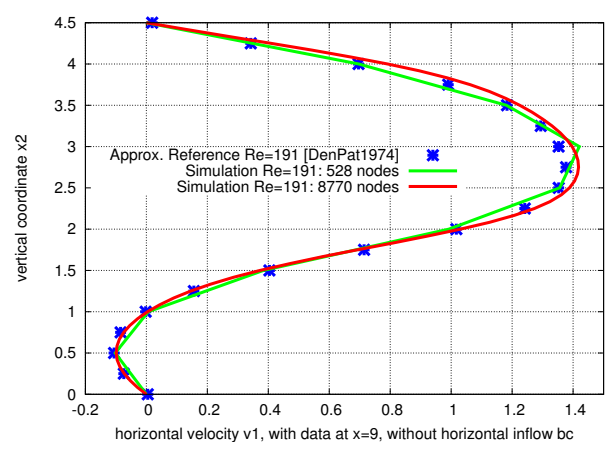

(b) $x=3$

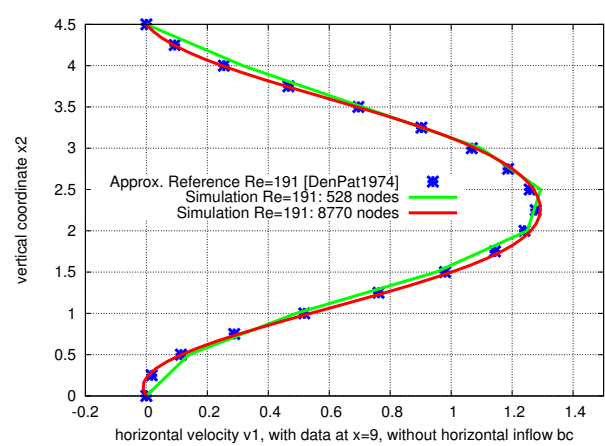

(d) $x=12$

Figure 9: Horizontal velocity profiles. No inflow condition specified, experimental data at $x_{d}=9$.

Note that these results are contingent on a reasonable choice of the weak BC weighting. Choosing the $\mathrm{BC}$ weighting too small will result the weak $\mathrm{BC}$ having negligible influence on the solution (giving the results of Section 4.4.1), too large and the inflow condition will be forced to match the (incorrect) black parabola in Figure 10 (a).

\section{Conclusions}

It has been shown how a modified LSFEM solver, combined with experimental data can:

- Correct the effects of discretization error,

- Correct inaccurate boundary conditions,

- Approximate fully unknown inflow conditions,

at a cost no greater than that of a standard LSFEM solve. In numerical experiments on a backward-facing step the introduction of experimental velocity profiles at one location has substantially improved the match with the experiment both up- and downstream. This has applications in many situations in which direct measurement of quantities of interest is impractical, but in which measurement of related quantities are easy. 


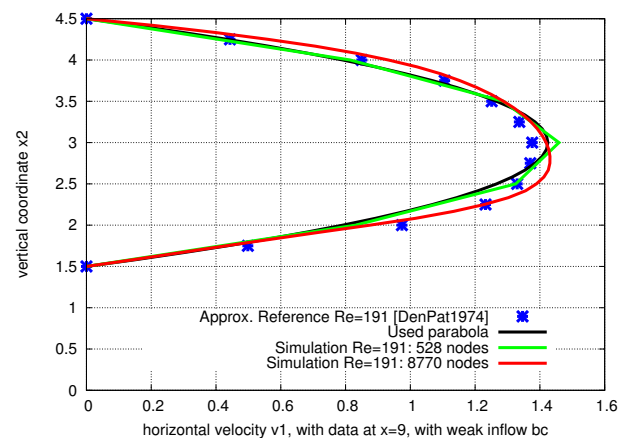

(a) $x=-2$

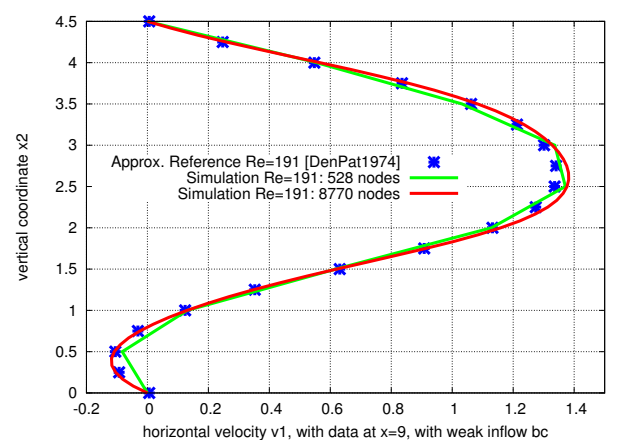

(c) $x=6$

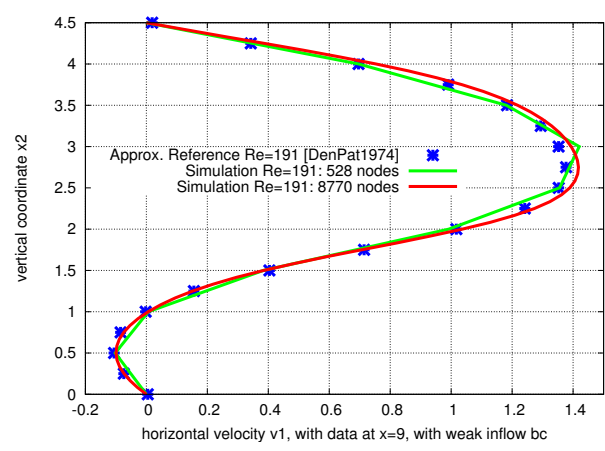

(b) $x=3$

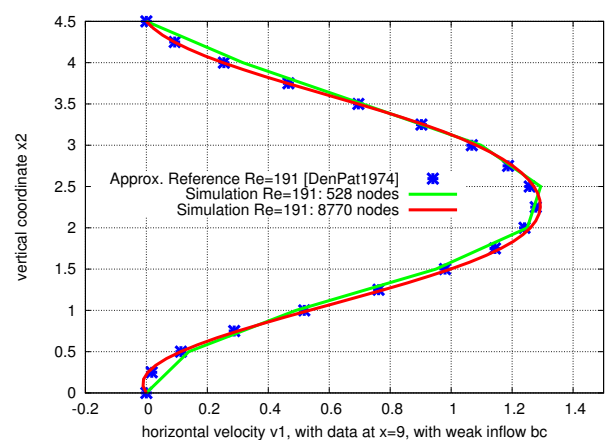

(d) $x=12$

Figure 10: Horizontal velocity profiles. Weakly enforced parabolic inflow, experimental data at $x_{d}=9$.

The number one obstacle to the adoption of this technique in practice, for example to augment gappy PIV data in the style of Sciacchitano et al. [3], is the limited capabilities of current LSFEM codes. To be useful in postprocessing experiments in incompressible aerodynamics the method must be able to treat high Reynolds numbers, use RANS turbulence modelling, with good boundary-layer resolution, low dissipation and little mass-loss. It is hoped that with further numerical developments such as the introduction of higher-order elements, improved first-order system choices, and local mesh refinement [50, 51] - applications of LSFEM to fluids will become more common. The approach described in this paper can nonetheless be employed for other physical problems where efficient LSFEM solvers already exist, e.g. in magnetohydrodynamics [52]. Furthermore the ability to assimilate experimental measurements at no additional cost, may make LSFEM competitive in applications in which it would not be competitive as a stand-alone solver.

\section{References}

[1] D. Ragni, PIV-based loads determination in aircraft propellers, Ph.D. thesis, TU Delft (2012).

[2] F. Scarano, P. Moore, An advection-based model to increase the temporal resolution of PIV time series, Experiments in Fluids 52 (2012) 919-933. 
[3] A. Sciacchitano, R. Dwight, F. Scarano, Navier-Stokes simulations in gappy PIV data, Experiments in Fluids.

[4] E. Constantinescu, M. Anitescu, Physics-based covariance models for Gaussian processes with multiple outputs, International Journal on Uncertainty Quantification.

[5] D. Ragni, B. van Oudheusden, F. Scarano, Non-intrusive aerodynamic loads analysis of an aircraft propeller blade, Experiments in Fluids 51 (2011) 361-371.

[6] B.-N. Jiang, The Least-Squares Finite Element Method: Theory and Applications in Computational Fluid Dynamics and Electromagnetics, 1st Edition, Springer, Berlin, 1998.

[7] P. B. Bochev, M. D. Gunzburger, Least-Squares Finite Element Methods, 1st Edition, Vol. 166 of Applied Mathematical Sciences, Springer, New York, 2009.

[8] J. Heys, T. Manteuffel, S. McCormick, M. Milano, J. Westerdale, M. Belohlavek, Weighted leastsquares finite elements based on particle imaging velocimetry data, Journal of Computational Physics 229 (1) (2010) 107-118. doi:doi:10.1016/j.jcp.2009.09.016

[9] R. Dwight, Bayesian inference for data assimilation using least-squares finite element methods, IOP Series: Materials Science and Engineering 10 (1) (2010) 222-232. doi:doi:10.1088/1757-899X/10/ $1 / 012224$

[10] Z. Cai, B. Lee, P. Wang, Least-squares methods for incompressible newtonian fluid flow: linear stationary problems, SIAM Journal on Numerical Analysis 42 (2004) 843-859.

[11] G. Evensen, The ensemble Kalman filter: Theoretical formulation and practical implementation, Ocean Dynamics 53 (2003) 343-367.

[12] G. Evensen, P. van Leeuwen, An ensemble Kalman smoother for nonlinear dynamics, Monthly Weather Review 128 (2000) 1852-1867.

[13] P. LeGresley, J. Alonso, Improving the Performance of Design Decomposition Methods with POD, American Institute of Aeronautics and Astronautics, 2004.

[14] R. Zimmermann, S. Grtz, Non-linear reduced order models for steady turbulent aerodynamics, Royal Aerospace Society Journal.

[15] T. Hayase, K. Imagawa, K. Funamoto, , A. Shirai, Stabilization of measurement-integrated simulation by elucidation of destabilizing mechanism, Journal of Fluid Science and Technology 5 (3) (2010) 632-647.

[16] K. Imagawa, T. Hayase, Eigenvalue analysis of linearized error dynamics of measurement integrated flow simulation, Computers and Fluids 39 (10) (2010) 1796-1803.

[17] B.-N. Jiang, The Least-Squares Finite Element Method, Springer-Verlag, Berlin, 1998.

[18] P. Bochev, M. Gunzburger, Least-Squares Finite Element Methods, 1st Edition, Springer-Verlag, New York, 2009.

[19] O. Kayser-Herold, H. Matthies, Least-squares FEM, literature review, Informatik-Bericht 2005-05, TU Braunschweig, Institut für Wissenschaftliches Rechnen.

[20] J. P. Pontaza, Least-squares variational principles and the finite element method: theory, form, and model for solid and fluid mechanics, Ph.D. thesis, Texas A\&M University (2003).

[21] B.-N. Jiang, C. Chang, Least-squares finite elements for the Stokes problem, Computer Methods in Applied Mechanics and Engineering 78 (1990) 297-311.

[22] P. Bochev, Analysis of least-squares finite element methods for the Navier-Stokes equations, SIAM Journal on Numerical Analysis 34 (1997) 1817-1844.

[23] B. Bell, K. Surana, p-version least squares finite element formulation for two-dimensional, incompressible, non-newtonian isothermal and non-isothermal fluid flow, International Journal for Numerical Methods in Fluids 18 (1994) 127-162.

[24] P. Bochev, M. Gunzburger, Least-squares methods for the velocity-pressure-stress formulation of the Stokes equations, Computer Methods in Applied Mechanics and Engineering 126 (1995) 267-287.

[25] X. Ding, T. Tsang, On first-order formulations of the least-squares finite element method for incompressible flows, International Journal of Computational Fluid Dynamics 17 (2003) 183-197.

[26] Z. Cai, T. Manteuffel, S. McCormick, First-order system least squares for the Stokes equation, with application to linear elasticity, SIAM Journal on Numerical Analysis 34 (1997) 1727-1741.

[27] P. Bochev, Z. Cai, T. Manteuffel, S. McCormick, Analysis of velocity-flux least-squares methods for the Navier-Stokes equations, part I, SIAM Journal on Numerical Analysis 35 (1998) 990-1009.

[28] P. Bochev, T. Manteuffel, S. McCormick, Analysis of velocity-flux least squares methods for the Navier-Stokes equations, part II, SIAM Journal on Numerical Analysis 36 (1999) 1125-1144.

[29] J. M. Deang, M. D. Gunzburger, Issues related to least-squares finite element methods for the Stokes equations, SIAM Journal on Scientific Computing 20 (3) (1998) 878-906.

[30] P. Bolton, R. W. Thatcher, On mass conservation in least-squares methods, Journal of Computational Physics 203 (2005) 287-304 
[31] C. L. Chang, J. J. Nelson, Least-squares finite element method for the Stokes problem with zero residual of mass conservation, SIAM Journal on Scientific Computing 34 (2) (1997) 480-489.

[32] J. P. Pontaza, A least-squares finite element form for unsteady incompressible flows with improved velocity-pressure coupling, Journal of Computational Physics 217 (2006) 563-588.

[33] J. P. Pontaza, J. N. Reddy, Spectral/hp least-squares finite element formulation for the NavierStokes equation, Journal of Computational Physics 190 (2003) 523-549.

[34] J. P. Pontaza, J. N. Reddy, Space-time coupled spectral/hp least-squares finite element formulation for the incompressible Navier-Stokes equation, Journal of Computational Physics 197 (2004) 418459 .

[35] M. M. J. Proot, M. I. Gerritsma, Mass- and momentum conservation of the least-squares spectral element method for the Stokes problem, Journal of Scientific Computing 27 (2006) 389-401.

[36] J. J. Heys, E. Lee, T. A. Manteuffel, S. F. McCormick, On mass-conserving least-squares methods, SIAM Journal on Scientific Computing 28 (3) (2006) 1675-1693.

[37] J. Heys, E. Lee, T. Manteuffel, S. McCormick, An alternative least-squares formulation of the navier-stokes equations with improved mass conservation, Journal of Computational Physics 226 (2007) 994-1006.

[38] J. Heys, E. Lee, T. Manteuffel, J. McCormick, S.and Ruge, Enhanced mass conservation in leastsquares methods for navier-stokes equations, SIAM J. Sci. Comp. 31 (2009) 2303-2321.

[39] O. Kayser-Herold, Least-squares methods for the solution of fluid-structure interaction problems, Dissertation, Technische Universität Braunschweig, Carl-Friedrich-Gauss-Fakultät für Mathematik und Informatik (2006).

[40] A. Schwarz, M. Nickaeen, S. Serdas, C. Nisters, A. Ouazzi, J. Schröder, S. Turek, A Comparative Study of Mixed Least Squares FEMs for the Incompressible Navier-Stokes Equations, International journal of Computational Science and Engineering in print.

[41] P. A. Raviart, J. M. Thomas, A mixed finite element method for 2-nd order elliptic problems. Mathematical aspects of finite element methods, Lecture Notes in Mathematics, Springer-Verlag New York (1977) 292-315.

[42] F. Brezzi, M. Fortin, Mixed and Hybrid Finite Element Methods, Springer-Verlag, New York, 1991.

[43] M. Kennedy, A. O'Hagan, Bayesian calibration of computer models (with discussion), Journal of the Royal Statistical Society, Series B. 63 (2001) 425-464.

[44] N. C. Nguyen, H. Men, R. M. Freund, J. Peraire, Functional regression for state prediction using linear pde models and observations, SIAM Journal on Scientific Computing 38 (2) (2016) B247B271. doi:10.1137/14100275X.

[45] M. Denham, M. Patrick, Laminar flow over a downstream-facing step in a two-dimensional flow channel, Transactions of the Institution of Chemical Engineers 52 (1974) 361-367.

[46] R. Barber, A. Fonty, A numerical study of laminar flow over a confined backward-facing step using a novel viscous-splitting vortex algorithm, in: Proc. 4th GRACM Congress on Computational Mechanics, Patra, 2002, pp. 1-8.

[47] J. de Baar, R. Dwight, H. Bijl, Fast maximum likelihood estimate of the kriging correlation range in the frequency domain, in: International Association for Mathematical Geosciences (IAMG) Conference, Salzburg, 2011.

[48] A. O'Hagan, Some Bayesian numerical analysis, Bayesian Statistics 4 (1992) 345-363.

[49] C. K. Wikle, L. M. Berliner, A Bayesian tutorial for data assimilation, Physica D: Nonlinear Phenomena $230(1-2)(2007) 1-16$

[50] R. Becker, R. Rannacher, Weighted a posteriori error control in FE methods, in: Proceedings of ENUMATH-97, Heidelberg, World Scientific Publishing, Singapore, 1998.

[51] R. Dwight, Heuristic a posteriori estimation of error due to dissipation in finite volume schemes and application to mesh adaptation, Journal of Computational Physics 227 (5) (2008) 2845-2863. doi:doi:10.1016/j.jcp.2007.11.020

[52] J. Adler, T. Manteuffel, S. McCormick, J. Ruge, First-order system least squares for incompressible resistive magnetohydrodynamics, SIAM J. Sci. Comput. (SISC) 32 (1) (2010) 229-248. 